\title{
Physiological suppression eases in Damaraland mole-rat societies when ecological constraints on dispersal are relaxed
}

\author{
ANDREW J. YOUNG ${ }^{1,2}$, MARIA K. OOSTHUIZEN ${ }^{3}$, HEIKE LUTERMANN $^{3}$ and \\ NIGEL C. BENNETT ${ }^{3}$
}

${ }^{1}$ Centre for Ecology and Conservation, University of Exeter, UK ${ }^{2}$ Department of Zoology, University of Cambridge, UK ${ }^{3}$ Department of Zoology and Entomology, University of Pretoria, South Africa

*Corresponding author:

Email: a.j.young@exeter.ac.uk; Telephone: +44-1326-254241; Fax: +44-1326-253638 Postal address: Centre for Ecology and Conservation, School of Biosciences, University of Exeter Cornwall Campus, Tremough, Cornwall TR10 9EZ, UK 


\section{ABSTRACT}

In many vertebrate societies, subordinate females exhibit down-regulated reproductive physiologies relative to those of dominants, a condition commonly termed physiological suppression. Research into the causes of physiological suppression has focused principally on the role of the subordinate's social environment (typically the presence of the dominant female and/or an absence of unrelated males within the group), while few studies have considered the additional role that the physical environment may play. Here we present new evidence from wild Damaraland mole-rats, Fukomys damarensis, revealing that physiological suppression among subordinate females eases markedly during the annual rains (a time when ecological constraints on dispersal are relaxed), despite the continued presence of the dominant female and in groups that contain no new immigrant males. Subordinate females showed substantially higher pituitary sensitivities to GnRH challenge during the wet period than the dry, a contrast that cannot be attributed to between-female differences (as it holds for paired within-female comparisons), associated changes in body mass (as our analyses control for this), or concomitant reductions in physiological stress (as their urinary cortisol concentrations were actually higher in the wet period). We suggest that our findings reflect selection for the maintenance of reproductive readiness among subordinate females during high rainfall periods, given the increased likelihood of encountering dispersal and/or mating opportunities with extra-group males when ecological constraints on dispersal are relaxed. These findings reveal new complexity in the processes that regulate physiological suppression, suggesting a key role in some species for changes in the physical as well as social environment.

KEYWORDS: reproductive conflict, reproductive suppression, reproductive skew, ecological constraints, rainfall, social stress, cooperative breeding, physiological suppression, Cryptomys damarensis 


\section{INTRODUCTION}

In many vertebrate societies, subordinate females breed at low rates and show downregulation at one or more levels of their reproductive endocrine axes relative to those of dominants, a condition commonly termed physiological suppression (Abbott et al. 1997; Bennett et al. 1999; Schoech et al. 2004; Young 2009). To date, research into the causes of physiological suppression among subordinates has focused principally on the likely importance of two key aspects of their social environment (reviewed in Young 2009). First, subordinate females commonly exhibit down-regulated reproductive physiologies when in the presence of the dominant female (e.g. Clarke et al. 2001; Saltzman et al. 2009). This may be due either to the subordinate exercising physiological restraint given a threat of interference by the dominant (Young et al. 2008; Saltzman et al. 2009) or to the dominant forcibly downregulating the subordinates' fertility by subjecting them to chronic stress (e.g. Young et al. 2006). Second, subordinate females commonly exhibit down-regulated reproductive physiologies when lacking unrelated mates within their groups, a condition that most likely reflects an inbreeding avoidance mechanism (e.g. Cooney and Bennett 2000; Carlson et al. 2004). Comparatively little work, however, has considered the extent to which subordinate females may also modulate their reproductive physiologies according to changes in their physical environment, which is perhaps surprising as such changes may markedly affect a subordinate's expected fitness payoff from maintaining their fertility.

In many social vertebrates, for example, a subordinate's reproductive potential is limited in large part by ecological constraints on extra-group movement, which reduce their chances of dispersing to independent breeding positions or encountering extra-group mates (Koenig et al. 1992; Hatchwell and Komdeur 2000; Young et al. 2007). Wherever variation in the strength of such ecological constraints does affect a subordinate's expected fitness payoff from maintaining their fertility, subordinates may be expected to modulate their reproductive 
physiology accordingly (favoring up-regulation when constraints are relaxed, to ensure reproductive readiness). Reproductive sensitivity to ecological constraints may therefore be at its most apparent in species that experience strong yet variable constraints on dispersal and independent reproduction.

One cooperative vertebrate that experiences acute yet variable constraints on dispersal is the Damaraland mole-rat, Fukomys damarensis (formerly known as Cryptomys damarensis; Kock et al. 2006). Damaraland mole-rats live in the deserts of southern Africa, where for much of the year the compacted-sand substrate through which they dig their foraging and dispersal tunnels is extremely costly to work (Lovegrove 1989); an ecological constraint that is thought to have favored the evolution of delayed dispersal (Jarvis and Bennett 1993; Jarvis et al. 1994; Faulkes et al. 1997). This constraint relaxes markedly, however, during periods of sustained rainfall, which soften the sands and stimulate prolific excavation and dispersal by subordinates of both sexes (to either found new colonies or, among males, to seek matings or immigration opportunities in other groups; Jarvis and Bennett 1993; Hazell et al. 2000; Burland et al. 2004). A subordinate female's expected fitness payoff from maintaining her fertility is likely to be much higher during such periods, not only because her own chances of successful dispersal are higher, but because her chances of encountering extra-group males with which to mate and/or disperse are also expected to increase (Jarvis and Bennett 1993; Hazell et al. 2000; Burland et al. 2004). In this study we therefore test the prediction that subordinate female Damaraland mole-rats, who exhibit marked physiological suppression (Bennett et al. 1999), should respond to the relaxation of ecological constraints associated with heavy rainfall by reducing the extent of their reproductive down-regulation.

Damaraland mole-rats live in colonies of 2-40 individuals in which the dominant female monopolizes reproduction and offspring of both sexes delay dispersal and help to rear the 
dominant's young (Jarvis and Bennett 1993). The complete suppression of subordinate female reproduction arises through a block on ovulation, which can be attributed to the downregulation of their pituitary sensitivity to Gonadotrophin Releasing Hormone (GnRH; Bennett et al. 1999; Molteno and Bennett 2000). The suppression of pituitary sensitivity to GnRH is mediated, at least in part, by two aspects of their social environment: the presence of the dominant female and a lack of unrelated males within the group. On removal from the dominant female, subordinate females show significant increases in pituitary sensitivity to GnRH and begin to ovulate spontaneously (Bennett et al. 1999; Molteno and Bennett 2000). The introduction of an unrelated male to a subordinate female housed with the dominant female, however, also leads to an increase in the subordinate's pituitary sensitivity to $\mathrm{GnRH}$, as well as copulations with the new male and extreme aggression among the females (Cooney and Bennett 2000).

While these social factors clearly play a key role in precipitating physiological suppression, there is limited evidence to suggest that subordinate females may also modulate their reproductive physiologies according to changes in their physical environment. A group of subordinate females collected during a wet period had higher pituitary sensitivities to GnRH than another collected during a dry period (Molteno and Bennett 2002). However, marked differences in body mass between the two groups, coupled with a lack of longitudinal data on their colonies, leave it possible that these physiological differences instead reflect betweenfemale differences in age and/or body condition or a response to recent male immigration.

In this study we use longitudinal data from a wild population of individually identifiable Damaraland mole-rats to investigate: (i) whether subordinate females up-regulate their reproductive physiologies during high rainfall periods (increasing either their baseline LH concentrations or pituitary sensitivity to GnRH); (ii) whether any such changes could be 
attributable simply to associated changes in body mass or social context; and (iii) whether any reproductive up-regulation detected could be due instead to a concomitant reduction in the extent of socially-induced stress experienced by subordinates (as evidenced by a reduction in their urinary cortisol concentrations). In addition, we investigate whether the focal high rainfall period does elicit the predicted increase in extra-group movement and dispersal.

\section{METHODS}

\section{Study population and trapping methods}

This study uses data arising from the longitudinal monitoring of Damaraland mole-rat colonies in the semi-arid wilderness of Tswalu Kalahari Reserve $\left(27^{\circ} 22^{\prime} \mathrm{S}, 22^{\circ} 19^{\prime} \mathrm{E}\right)$, in the Northern Cape province of South Africa, between 2004 and 2006. The study site lies in a wide basin of thorny Kalahari bushveld, sparsely vegetated with grasses (Eragrostis, Stipagrostis, Aristade, and Schmidtia species), bushes (Acacia mellifera, Rhigozum trichotomum, Grewia flava and Lycium cinereu) and trees (Acacia erioloba, Acacia haematoxylon and Boscia albitrunca). The average annual rainfall over the period 2001 to 2008 was $297 \mathrm{~mm}$ (range 153-459 mm), much of which fell in a limited number of heavy storms unpredictably distributed throughout the hotter summer months (October to April; average maximum daytime temperature $=35.2^{\circ} \mathrm{C}$; average monthly rainfall $=39.6 \mathrm{~mm}$ ), while comparatively little fell during the winter (May to September; average maximum daytime temperature $=25.1^{\circ} \mathrm{C}$; average monthly rainfall $=7.1 \mathrm{~mm}$ ). We contrasted the physiological parameters of mature subordinate females caught during a trapping session towards the end of a prolonged dry period $\left(1^{\text {st }}\right.$ to $15^{\text {th }}$ October 2004; Figure 1$)$ with those of mature subordinate females caught during a trapping session at the peak of the subsequent wet period $\left(20^{\text {th }}\right.$ March to $17^{\text {th }}$ April 2005; Figure 1). 
Colonies were trapped by digging a short trench across a line of mole-hills to locate the underlying tunnel (typically at a depth of 20 to $80 \mathrm{~cm}$ ) and then setting pipe traps baited with sweet potato in the line of the tunnel. Traps were typically checked every 1-3 hours and any trapped animals immediately transferred to spacious individual urine collection traps (see below) and weighed using an electronic balance (Ohaus CS2000). Once a urine sample had been passed (or if nothing had been passed for $>4$ hours), the animals were transferred to a large sand-lined box for housing with the other members of their colony and were sustained on sweet potato. Once the colony had been completely trapped out (gauged by an absence of activity at the trap site for 36 hours) and urine and blood sampling had been completed, any unmarked animals (distinguished until now by their sex, weight and coat patterns) were marked with subcutaneous transponder chips and the entire colony was returned together to their original tunnel system.

A colony was defined as the group of one or more individuals caught from the same contiguous burrow system. Typically all colony members were caught from a single trap site, but occasionally two distant trap sites were found to be catching from the same colony, as highlighted by our knowledge of prior colony compositions and/or the absence of a dominant breeding female in one half, and confirmed in each case by the release of individuals at one of the trap sites and their subsequent capture at the other. The captured colonies comprised an average (range) of 6.3 (1-24) animals of approximately equal sex ratio, with males weighing an average (range) of 104.8 (16-215) g and females weighing an average (range) of 98.5 (28171) g. The dominant female in each colony (of which there was never more than one) could be readily distinguished from her non-breeding subordinates by her perforate vagina and/or swollen teats (Bennett and Faulkes 2000; as validated by Burland et al. 2004), and the sexes were differentiated by the shape of their genitalia (Bennett and Faulkes 2000). All protocols 
were approved by the University of Pretoria ethics committee and complied with regulations stipulated in the Guidelines for the use of Animals in Research.

\section{Blood sampling, GnRH administration and LH bioassay}

To determine pituitary sensitivity to $\mathrm{GnRH}$, we conducted GnRH challenges as follows: we first collected a blood sample for baseline LH level determinations, then administered the GnRH challenge, and collected a second blood sample 20 minutes later for post GnRHchallenge LH level determinations. Blood samples $(\sim 200 \mu 1)$ were collected by briefly restraining the animals in a soft towel, piercing a foot vein with a sterile needle and drawing blood from it using a heparinized microhaematocrit tube. The samples were kept cool at $\sim 4^{\circ} \mathrm{C}$ for up to $20 \mathrm{~min}$ until they could be centrifuged at $500 \mathrm{~g}$ and their plasma drawn off and stored at $-25^{\circ} \mathrm{C}$ until assay. The $\mathrm{GnRH}$ challenge took the form of a single subcutaneous injection of $2 \mu \mathrm{g}$ exogenous GnRH in $200 \mu$ l of physiological saline (following Bennett et al. 1993). The GnRH was synthesized in the laboratory of R. P. Millar (the purity of GnRH was greater than 98\% homogeneity; Millar et al. 1989). To minimize diel variation in hormone levels and pituitary responses, all blood sampling was conducted between 17:00 and 20:00 on the day following capture.

Plasma concentrations of $\mathrm{LH}$ were determined for both baseline and post-GnRH samples using an in vitro bioassay based on the production of testosterone by dispersed mouse Leydig cells (Van Damme et al. 1974), as previously described and validated for Damaraland molerats (Bennett et al. 1993; Molteno and Bennett 2002). Plasma samples were assayed in duplicate at a dilution of 1:20 and compared with a rat LH standard (the rLH antigen preparation: rLH-1-7 from NIDDK Baltimore) over the range 0.031-400 $\mathrm{miu} / \mathrm{ml}$. The testosterone produced was then measured by radioimmunoassay as described by Hodges et al. 
(1987). Assay sensitivity (determined at $90 \%$ binding) was $0.1 \mathrm{miu} /$ tube and intra- and interassay coefficients of variation were $6.1 \%$ and $11.2 \%$ respectively.

\section{Urine sample collection and urinary cortisol assay}

To assess changes in the stress physiology of subordinate females, we collected naturally passed urine samples as soon as possible following capture. Trapped animals were immediately placed in individual urine traps, in which they rested on a wire mesh through which urine could pass into a collection reservoir below. The urine traps were checked every 20-30 minutes and any urine was immediately collected and stored on ice until it could be transferred (within 3 hours) to a $-25^{\circ} \mathrm{C}$ freezer for storage until assay. Samples were collected over a range of times of day (09:30 to 21:00), but we later controlled for any circadian variation in cortisol concentrations in our statistical models, along with variation in the time lag between capture and sample collection.

Cortisol assays were performed using a coat-a-count cortisol kit (Diagnostic Products Corporation) that had previously been validated for urinary cortisol monitoring in Damaraland mole-rats (Clarke et al. 2001). All samples were assayed in duplicate and the assay sensitivity (determined at $90 \%$ binding) was $6.1 \mathrm{ng} / \mathrm{ml}$. The intra-assay coefficient of variation was $5.5 \%$ (the samples were run in a single assay). To correct for variation in urinary cortisol concentrations resulting from variation in urine concentration, determinations of creatinine concentrations were also conducted in duplicate for all urine samples (as described by Bonney et al. 1982; Clarke et al. 2001). The intra-assay coefficient of variation was 3.0\%. All urinary cortisol concentrations were subsequently expressed as nMol cortisol / nMol creatinine. 


\section{Statistical analysis of endocrine data}

All statistical tests were two-tailed and conducted using GenStat 10 (Lawes Agricultural Trust, Rothamsted, UK). To ensure that our comparisons of subordinate female traits across the two periods were not complicated by the inclusion of non-reproductive-age females (juveniles), our analyses included only those females whose body mass exceeded 88g (the mass of the lightest breeding female trapped in this population). Comparisons of the physiological parameters of subordinate females between the wet period and the dry (specifically their baseline and post-GnRH plasma LH concentrations and their urinary cortisol concentrations) were conducted using General Linear Mixed Models (GLMMs). These are similar to General Linear Models except that they allow both fixed and random terms to be defined, the latter being used in this case to control in each model for repeated measures of groups and individuals.

To identify any changes in the reproductive physiologies of subordinate females between the dry and wet periods, we conducted three statistical tests. First, we tested whether females' LH concentrations following GnRH challenge differed between the two periods, by fitting period (dry or wet) in a GLMM of factors affecting post-GnRH LH levels, with body mass fitted as a covariate. To then determine whether any increase in post-GnRH LH levels was attributable in part to an increase in pituitary sensitivity to the GnRH challenge (rather than an increase in baseline LH), we tested the significance of the interaction between period (dry or wet) and challenge (baseline LH or post-challenge LH) in a GLMM of factors affecting plasma LH levels, again fitting body mass as a covariate. Finally, we tested whether baseline LH concentrations had also increased between the dry and wet periods, by fitting period (dry or wet) in a GLMM of factors affecting baseline LH levels, with body mass fitted as a covariate. Both colony and individual identity were fitted as random factors in all three models. These analyses were based on a total sample of 66 plasma LH determinations (33 baseline and 33 
post-GnRH) for 20 subordinate females captured from 9 colonies during the dry period and 13 subordinate females captured from 7 colonies during the wet period. To establish whether our findings could be attributable to differences between the females sampled in each period, we also conducted paired within-female comparisons between the two periods, for post-GnRH LH levels, pituitary responses to GnRH challenge (post-GnRH LH - baseline LH), and baseline LH levels, using only the 11 subordinate females that were sampled in both conditions.

To test whether any reproductive up-regulation detected during the wet period could be due to a concomitant reduction in the extent of physiological stress experienced by subordinates, we investigated whether females' urinary cortisol concentrations differed between the two periods. Period (dry or wet) was fitted as a fixed effect in a GLMM of factors affecting the females' log-transformed urinary cortisol concentrations, along with the following covariates: body mass; the time of sample collection during the day (both hour of the day and hour ${ }^{2}$ were fitted to allow for non-linear circadian variation); and the maximum possible time lag between capture and urine sample collection. The latter was fitted to control for the increases in urinary cortisol concentrations that are expected to follow capture. This 'maximum time lag' was calculated as the time lag between the time the trap was last checked prior to capture and the time the urine sample was collected (the actual time lag between capture and sampling will always be slightly shorter than this). To allow for the possibility that the nature of the stress response to capture varied between the periods, we also tested for an interaction between the period (dry or wet) and the maximum possible time lag from capture. The analysis was based on 33 urine samples collected from 14 subordinate females from 9 colonies in the dry period and 15 subordinate females from 12 colonies in the wet period. All urine samples were collected within a maximum possible time lag between capture and sampling of 10 hours. 


\section{Dispersal analyses}

To investigate whether the incidence of dispersal differed between the dry and wet periods, we drew upon several lines of evidence. First, we compared the change in colony size distribution during the dry period to that during the wet period. We predicted that, if significant dispersal occurred during the wet period, the average colony size should fall over the course of the wet period, while no such fall would be expected during the dry period. To determine the change in average colony size over the dry period we compared colony sizes during a trapping session at the start of the dry period $\left(20^{\text {th }}\right.$ March to $17^{\text {th }}$ April 2004; a trapping session not yet discussed as no physiological measures were taken) to those at the end of the dry period (the key dry period trapping session already discussed; Figure 1). To determine whether average colony size fell over the course of the wet period we compared colony sizes at the end of the dry period (Figure 1) to those at the peak of the subsequent wet period (the key wet period trapping session already discussed; Figure 1). For each comparison we used all colonies trapped during that session for which colony size (the number of individuals in the colony) was accurately known (i.e. when we were able to trap until there had been no activity within the trap site for $>36$ hours). To establish whether the reduction in average colony size detected over the course of the wet period could be attributed at least in part to dispersal (rather than within-group mortality), we also tested whether the proportion of colonies that comprised 'probable dispersers' increased over the course of the wet period. Colonies were considered to comprise probable dispersers if they contained only adults who had not previously been trapped away from an established colony and were currently either alone, in a pair (mixed or same-sex) or a mixed sex trio (same-sex dispersing pairs have been reported elsewhere and noted within this study population; Bennett and Faulkes 2000). 


\section{RESULTS}

\section{Reproductive physiology}

The rainfall leading up to the captures at the peak of the wet period was markedly higher than that leading up to the captures at the end of the dry period (Figure 1). As predicted, there was an associated up-regulation of the reproductive physiologies of subordinate females during the wet period (Figure 2), despite the sampled females remaining within the same groups, alongside the same dominant females and their groups having been joined by no new immigrant males. Subordinate adult females exhibited markedly higher post GnRH-challenge LH concentrations during the wet period captures than the dry, due principally to a significantly stronger effect of the GnRH challenge on circulating LH concentrations during the wet period (Figure 2). These analyses controlled for variation among females in body mass (see Figure 2 legend), though the sampled females did not differ significantly in body mass between the two periods (Mann-Whitney $\mathrm{U}=122.0 ; \mathrm{P}=0.78 ; \mathrm{n}=20,13$; dry period median (Inter-Quartile Range (IQR)) mass = 101.9 (92.5-114.6) g; wet period median (IQR) mass = $106.0(88.8-114.5) \mathrm{g})$.

Paired within-female comparisons using the subset of subordinate females that were sampled in both periods ( $\mathrm{n}=11)$ confirm each of these findings (Table 1). The subordinate females exhibited significantly higher post GnRH-challenge LH concentrations in the wet period relative to the dry, attributable principally to a significantly stronger effect of the challenge on LH levels in the wet period than in the dry (Table 1). There was no significant increase in the females' baseline LH concentrations between the two periods, nor did the subordinate females significantly change in body mass (Table 1). In accordance with previous assertions that Damaraland mole-rats are aseasonal breeders, established colonies were equally likely to contain pups (individuals $<40 \mathrm{~g}$ ) in the dry period (4 of 10 established colonies; $40.0 \%$ ) and the wet ( 7 of $17 ; 41.2 \%$; Fisher's exact $\mathrm{P}=1.00)$. 


\section{Stress physiology}

The reproductive physiological up-regulation of subordinate females during the wet period was not associated with a reduction in urinary cortisol concentrations. On the contrary, subordinate female urinary cortisol concentrations were substantially higher during the wet period than the dry (Figure 3a; GLMM: $\chi^{2}{ }_{1}=12.63$; $\mathrm{P}=0.005$; while controlling for a positive effect of the time lag from capture to sampling: Figure $\left.3 b ; \chi_{1}^{2}=9.84 ; \mathrm{P}=0.004\right)$. Urinary cortisol levels were not correlated with body mass $\left(\chi_{1}^{2}=2.60 ; \mathrm{P}=0.12\right)$ and the inclusion of body mass in the model had no qualitative effect on the significance of the other terms. There was no significant interaction between period (wet or dry) and time since capture $\left(\chi_{1}^{2}=0.14\right.$; $\mathrm{P}=0.71$; Figure $3 \mathrm{~b}$ ), suggesting that there was no marked difference between the periods in the urinary manifestation of the stress response to capture. There was no significant circadian variation in urinary cortisol concentrations over the range of our sampling times (Hour effect: $\chi_{1}^{2}=0.24 ; \mathrm{P}=0.63 ;$ Hour $^{2}$ effect: $\left.\chi^{2}{ }_{1}=0.14 ; \mathrm{P}=0.71\right)$.

\section{Dispersal patterns}

As predicted, our findings suggest that the wet period triggered a marked increase in dispersal activity. Over the five drier months leading up to the captures at the end of the dry period, the average colony size in our study population did not change significantly (median at the start of the dry period $=9.0($ IQR $5.5-11.5)$ individuals; median at the end of the dry period $=8.5$ (3.0-10.5) individuals; $\mathrm{n}=21,12$ colonies; $\mathrm{U}=98.0 ; \mathrm{P}=0.30$ ). By contrast, over the subsequent five wetter months leading up to the captures at the peak of the wet period, the average colony size decreased significantly (median at the end of the dry period $=8.5$ (IQR 3.0-10.5) individuals; median at the peak of the wet period $=3.0$ (IQR 1.5-6.0) individuals; $n=12,36$ colonies, $\mathrm{U}=118.5 ; \mathrm{P}=0.018$ ). This reduction in average colony size was due principally to a substantial increase in the proportion of burrow systems occupied by probable dispersers, 
rising from 2 of 12 burrows (16.7\%) at the end of the dry period to 19 of 36 burrows $(52.8 \%)$ at the peak of the wet period (Fisher's exact $\mathrm{P}=0.044$ ). Five of these probable wet period dispersers (all females captured alone), are known with certainty to have dispersed as they had previously been trapped as subordinates within established colonies elsewhere; they had dispersed straight-line distances of $423 \mathrm{~m}, 370 \mathrm{~m}, 278 \mathrm{~m}, 133 \mathrm{~m}$ and $41 \mathrm{~m}$ between trap sites (the median nearest-neighbor distance between the trap sites of different colonies was $52 \mathrm{~m}$ (IQR 31-73m)). In addition, during the wet period trapping session, members of one established colony broke through into the burrow system of another, and on two occasions a singleton male entered another colony's burrow system (having dispersed $233 \mathrm{~m}$ and $147 \mathrm{~m}$ from their original colonies, where they had been trapped three weeks earlier). One of these intruder males subsequently founded a new colony with a subordinate female from the colony he had visited, while the other was never seen again.

\section{DISCUSSION}

Our findings reveal that subordinate female Damaraland mole-rats experienced a marked upregulation of their pituitary sensitivities to GnRH (the suppression of which is thought to underpin anovulation; Molteno and Bennett 2000) during the wet period relative to the dry. These physiological changes are comparable in nature to those elicited in laboratory studies by changing either of the two aspects of the social environment currently thought to regulate physiological suppression among subordinates (the removal of the dominant female or the insertion of an unrelated male into the group; Molteno and Bennett 2000; Cooney and Bennett 2000). Without exception, however, the sampled subordinates remained within the same groups across the two periods, alongside the same dominant females, and their groups contained no new immigrant males when trapped in the wet period. These physiological changes cannot, therefore, be readily attributed to permanent changes in either aspect of their social environment. 
The increase in pituitary sensitivity to GnRH during the wet period cannot be readily attributed to among-female differences across the two periods, as it remains significant in paired within-female comparisons. It is not likely either that these physiological contrasts reflect the reproductive maturation of pre-reproductive-age females between the two periods, as the sampled females lay within the known body mass range of breeding dominants and did not increase significantly in body mass between the two periods (in either the full or withinfemale analyses). The absence of a change in body mass, coupled with the fact that our statistical models controlled for variation in body mass, makes it unlikely too that these physiological differences reflect a response to improved body condition in the wet period (a factor known to alleviate physiological down-regulation among subordinates in another cooperative mammal; Young et al. 2008). It also seems unlikely that this contrast reflects a signature in subordinates of a seasonal pattern of reproduction in the species, as our finding that young offspring were equally likely to be found in colonies in the wet and dry periods accords with previous assertions that this species is an aseasonal breeder (Bennett and Faulkes 2000).

Reproductive physiological changes among subordinates might alternatively arise from changes in the extent to which the dominant is forcibly down-regulating their fertility by subjecting them to chronic stress (e.g. Young et al. 2006). However, our findings suggest that the observed reproductive up-regulation during the wet period cannot be attributed to a concomitant reduction in socially-induced stress, as subordinates' urinary cortisol concentrations were not lower in the wet period than the dry; in fact the opposite was true. As our findings control for variation in the capture-to-sampling time lag and allow for the possibility that the shape of the stress response to capture differed across the two periods, the documented increase in urinary cortisol concentrations most likely reflects an increase in 
baseline plasma cortisol concentrations during the wet period, which could have one of several causes. The increase could simply reflect the energetic demands associated with the wet period surges in tunneling (Jarvis and Bennett 1993) or the stimulatory effect that increases in circulating LH levels, and/or consequent increases in estrogen levels, can have on the hypothalamic-pituitary-adrenal axis (see Saltzman et al. 2006a; 2006b and discussion therein). More intriguing, however, is the possibility that this contrast could also reflect an increase in social stress induced by the dominant female, who may be selected to respond aggressively either to physiological up-regulation among subordinate females or to environmental conditions conducive to forcing their dispersal (Young et al. 2006; Stephens et al. 2005). As such, attempts by future studies to identify the proximate cause(s) of this marked $(\sim 140 \%)$ increase in urinary cortisol concentrations could yield valuable new insights into the nature of social conflict in wild mole-rat societies.

That physiological suppression among subordinate female Damaraland mole-rats eases when ecological constraints on dispersal are relaxed might initially be considered evidence in support of a central yet rarely tested prediction of concession models of reproductive skew. In such models the dominant is commonly envisaged to concede that share of reproduction to subordinates that is necessary to retain them within the group (Keller and Reeve 1994; Johnstone 2000). A key prediction, therefore, is that dominants should become more tolerant of subordinate reproduction when ecological constraints on dispersal are relaxed, because the subordinate's expected fitness payoff from departure rises. It is highly unlikely, however, that our findings reflect the outcome of such a social contract over reproduction. First, as subordinate female Damaraland mole-rats never breed (Molteno and Bennett 2000; Burland et al. 2004), they only stand to benefit from such reproductive physiological up-regulation if they disperse or challenge the dominant (contra to the logic of concession theory). Second, if this up-regulation was 'conceded' by a dominant with complete reproductive control, one 
might expect it to arise from a reduction in dominant-imposed physiological stress among subordinates, yet our findings indicate the contrary. Like many of the reproductive patterns in vertebrate societies that initially appear consistent with concessions approaches to reproductive skew, a more plausible explanation exists for our findings that does not require the invocation of reproductive contracts between dominants and subordinates (Clutton-Brock 1998; Magrath et al. 2004).

The most plausible explanation for reproductive up-regulation among subordinate females during the wet period would seem to be that their likelihood of encountering viable dispersal and/or mating opportunities with extra-group males increases markedly during such times, leading to selection for the maintenance of reproductive readiness to exploit such opportunities should they then arise. As morphological and genetic evidence suggest that female Damaraland mole-rats never rear litters as subordinates (Molteno and Bennett 2000; Burland et al. 2004), encounters with dispersing extra-group males are unlikely to offer females an opportunity to reproduce while remaining subordinate as has been reported for other social vertebrates (e.g. Young et al. 2007). Instead, such males constitute potential partners with whom to either disperse and found a new group or mate prior to dispersing alone. Should such extra-group males immigrate permanently, however, they would also provide subordinate females with an opportunity to become the resident breeder were they to challenge the dominant (Cooney and Bennett 2000; Hazell et al. 2000). In addition to maintaining reproductive readiness to exploit such opportunities, the observed reproductive physiological up-regulation could also play a key role in any sexual signaling necessary for securing a mate or dispersal partner during such rare encounters (see Spinks et al. 2000).

While the observed reproductive up-regulation certainly coincides with the high rainfall period, several plausible candidates exist for the cues that elicit these changes. It seems most 
likely that females respond directly to the fallen rain, by perceiving either a moistening of the sand in their tunnel systems or a change in burrow humidity; the same cues that are thought to stimulate excavation following heavy rain (Bennett and Faulkes 2000). If the payoff from these physiological changes does derive, as we suggest, from the relaxation of dispersal constraints during high rainfall periods, selection would be expected to favor a response to rainfall per se over other seasonal cues, such as temperature or even photoperiod, that only poorly predict the timing and extent of rainfall in this region. While the sampled females' groups contained no new immigrant males when trapped during the wet period, we cannot rule out any role for social cues in eliciting the observed physiological changes. Without the ability to continuously monitor intrusion into burrow systems, for example, it is difficult to exclude the possibility that these changes could also reflect a relic of responses to prior intrusions by extra-group males who did not remain in the colony. It does seem unlikely though that all sampled females had encountered intruding males for long enough to stimulate these physiological changes, and that all such males then left prior to trapping without these females joining them. Evidence of seismic communication in this species (by means of footdrumming; Bennett and Jarvis 1988b) does, however, raise the further possibility of a role for social cues from extra-group males without the need for them to enter the females' colonies.

Together, our findings suggest that physiological suppression among subordinate females in Damaraland mole-rat societies is not a stable year-round phenomenon mediated solely by the presence of the dominant female and an absence of permanent immigrant males. While it is clear from laboratory studies that such factors play a key role (Bennett et al. 1996; Cooney and Bennett 2000; Clarke et al. 2001), our longitudinal data suggest that subordinate females in wild populations also modulate their reproductive physiology according to the strength of ecological constraints on dispersal and/or associated changes in the strength of social cues from extra-group males. The physiological changes documented here also highlight the 
possibility of a seasonal component to reproductive conflict among females in Damaraland mole-rat societies, with dominants potentially facing threats associated with periodic reproductive up-regulation among subordinates (e.g. Young and Clutton-Brock 2006), to which they may respond by inducing physiological stress. Studies of the causes of physiological suppression in vertebrate societies may therefore benefit more broadly from considering the role that ecological changes may play, in addition to permanent changes in social structure, in modulating reproductive function among subordinates. Our findings lend strength to the emerging consensus that physiological suppression among subordinate vertebrates commonly arises from reproductive restraint exercised by subordinates in response to a suite of factors that act in concert to reduce their expected fitness payoff from maintaining their fertility (Young et al. 2008; Young 2009).

\section{ACKNOWLEDGMENTS}

We thank the host of volunteers who have provided invaluable field assistance, E. Oppenheimer \& Son and the staff of Tswalu Kalahari Reserve for permission to work on the reserve and the provision of exceptional logistical support, Northern Cape Conservation for permission to conduct the study, and the Association for the Study of Animal Behaviour, the Natural Environment Research Council, Magdalene College, Cambridge and the National Research Foundation for funding our research. 


\section{REFERENCES}

Abbott, D.H., Saltzman, W., Schultz-Darken, N.J., Smith, T.E., 1997. Specific neuroendocrine mechanisms not involving generalized stress mediate social regulation of female reproduction in cooperatively breeding marmoset monkeys. Ann NY Acad Sci 807, 219-238.

Bennett, N.C., Faulkes, C.G., 2000. African mole-rats: ecology and eusociality. Cambridge: Cambridge University Press.

Bennett, N.C., Faulkes, C.G., Jarvis, J.U.M., 1999. Socially induced infertility, incest avoidance and the monopoly of reproduction in cooperatively breeding African molerats: Family Bathyergidae. Adv. Stud. Behav. 28, 75-114.

Bennett, N.C., Faulkes, C.G., Molteno, A.J., 1996. Reproductive suppression in subordinate, non-breeding female Damaraland mole-rats: two components to a lifetime of socially induced infertility. Proc. R. Soc. B 263, 1599-1603.

Bennett, N.C., Jarvis, J.U.M., 1988a. The reproductive biology of the Cape mole-rat, Georychus capensis (Rodentia, Bathyergidae). J. Zool. 214, 95-106.

Bennett, N.C., Jarvis, J.U.M., 1988b. The social structure and reproductive biology of colonies of the mole-rat, Cryptomys damarensis (Rodentia, Bathyergidae). J. Mammal. 69, 293-302.

Bennett, N.C., Jarvis, J.U.M., Faulkes, C.G., Millar, R.P., 1993. LH responses to single doses of exogenous GnRH by freshly captured Damaraland mole-rats, Cryptomys damarensis. J. Reprod. Fertil. 99, 81-86.

Bonney, R.C., Wood, D.J., Kleiman, D.G., 1982. Endocrine correlates of behavioral estrus in the female giant panda (Ailuropoda melanoleuca) and associated hormonal changes in the male. J. Reprod. Fertil. 64, 209-215.

Burland, T.M., Bennett, N.C., Jarvis, J.U.M., Faulkes, C.G., 2004. Colony structure and parentage in wild colonies of co-operatively breeding Damaraland mole-rats suggest incest avoidance alone may not maintain reproductive skew. Mol. Ecol. 13, 23712379 .

Carlson, A.A., Young, A.J., Russell, A.F., Bennett, N.C., McNeilly, A.S., Clutton-Brock, T., 2004. Hormonal correlates of dominance in meerkats (Suricata suricatta). Horm. Behav. 46, 141-150.

Clarke, F.M., Miethe, G.H., Bennett, N.C., 2001. Reproductive suppression in female Damaraland mole-rats Cryptomys damarensis: dominant control or self-restraint? Proc. R. Soc. B 268, 899-909. 
Clutton-Brock, T.H., 1998. Reproductive skew, concessions and limited control. Trends Ecol. Evol. 13, 288-292.

Cooney, R., Bennett, N.C., 2000. Inbreeding avoidance and reproductive skew in a cooperative mammal. Proc. R. Soc. B 267, 801-806.

Faulkes, C.G., Bennett, N.C., Bruford, M.W., Obrien, H.P., Aguilar, G.H., Jarvis, J.U.M., 1997. Ecological constraints drive social evolution in the African mole-rats. Proc. R. Soc. B 264, 1619-1627.

Hatchwell, B.J., Komdeur, J., 2000. Ecological constraints, life history traits and the evolution of cooperative breeding. Anim. Behav. 59, 1079-1086.

Hazell, R.W.A., Bennett, N.C., Jarvis, J.U.M., Griffin, M., 2000. Adult dispersal in the cooperatively breeding Damaraland mole-rat (Cryptomys damarensis): a case study from the Waterberg region of Namibia. J. Zool. 252, 19-25.

Hodges, J.K., Cottingham, P., Summer, P.M., Yingnan, L., 1987. Controlled ovulation in the marmoset monkey (Callithrix jacchus), with human chorionic gonadotropin following prostaglandin induced luteal regression. Fertility and Sterility 48, 299-305.

Jarvis, J.U.M., Bennett, N.C., 1993. Eusociality has evolved independently in 2 genera of Bathyergid mole-rats, but occurs in no other subterranean mammal. Behav. Ecol. Sociobiol. 33, 253-260.

Jarvis, J.U.M., Oriain, M.J., Bennett, N.C., Sherman, P.W., 1994. Mammalian eusociality - a family affair. Trends Ecol. Evol. 9, 47-51.

Johnson, M.H., Everitt, B.J., 1999. Essential Reproduction, Fifth Edition ed. Oxford: Blackwell Science Ltd.

Johnstone, R.A., 2000. Models of reproductive skew: a review and synthesis (invited article). Ethology 106, 5-26.

Johnstone, R.A., Cant, M.A., 1999. Reproductive skew and the threat of eviction: a new perspective. Proc. R. Soc. B 266, 275-279.

Keller, L., Reeve, H.K., 1994. Partitioning of reproduction in animal societies. Trends Ecol. Evol. 9, 98-102.

Kock, D., Ingram, C.M., Frabotta, L.J., Honeycutt, R.L., Burda, H., 2006. On the nomenclature of Bathyergidae and Fukomys n. gen. (Mammalia : Rodentia). Zootaxa $1142,51-55$.

Koenig, W.D., Pitelka, F.A., Carmen, W.J., Mumme, R.L., Stanback, M.T., 1992. The evolution of delayed dispersal in cooperative breeders. Q. Rev. Biol. 67, 111-150. 
Lovegrove, B.G., 1989. The cost of burrowing by the social mole-rats (Bathyergidae) Cryptomys damarensis and Heterocephalus glaber: the role of soil moisture. Physiol. Zool. 62, 449-469.

Magrath, R.D., Johnstone, R.A., Heinsohn, R.G., 2004. Reproductive skew. In: Ecology and evolution of cooperative breeding in birds (Koenig, W.D., Dickinson, J.L., eds). Cambridge: Cambridge University Press.

Millar, R.P., Flanagan, C.A., de Milton, R.C., King, J.A., 1989. Chimeric analogues of vertebrate gonadotropin releasing hormones comprising substitutions of the variant amino acids in positions 57 and 8. J. Biol. Chem. 264, 21007-21013.

Molteno, A.J., Bennett, N.C., 2000. Anovulation in non-reproductive female Damaraland mole-rats (Cryptomys damarensis). J. Reprod. Fertil. 119, 35-41.

Molteno, A.J., Bennett, N.C., 2002. Rainfall, dispersal and reproductive inhibition in eusocial Damaraland mole-rats (Cryptomys damarensis). J. Zool. 256, 445-448.

Saltzman, W., Digby, L.J., Abbott, D.H., 2009. Reproductive skew in female common marmosets: what can proximate mechanisms tell us about ultimate causes? Proc. R. Soc. B 276, 389-399.

Saltzman, W., Hogan, B.K., Allen, A.J., Horman, B.M., Abbott, D.H., 2006a. Hypoestrogenism does not mediate social suppression of cortisol in subordinate female marmosets. Psychoneuroendocrinology 31, 692-702.

Saltzman, W., Hogan, B.K., Horman, B.M., Abbott, D.H., 2006b. Social suppression of cortisol in female marmosets: Role of luteinizing hormone/chorionic gonadotropin. Gen. Comp. Endocrinol. 149, 90-99.

Schoech, S.J., Reynolds, S.J., Boughton, R.K., 2004. Endocrinology. In: Ecology and evolution of cooperative breeding in birds (Koenig, W.D., Dickinson, J.L., eds). Cambridge: Cambridge University Press; 128-141.

Spinks, A.C., Bennett, N.C., Faulkes, C.G., Jarvis, J.U.M., 2000. Circulating LH levels and the response to exogenous GnRH in the common mole-rat: implications for reproductive regulation in this social, seasonal breeding species. Horm. Behav. 37, 221-228.

Stephens, P.A., Russell, A.F., Young, A.J., Sutherland, W.J., Clutton-Brock, T.H., 2005. Dispersal, eviction, and conflict in meerkats (Suricata suricatta): An evolutionarily stable strategy model. Am. Nat. 165, 120-135. 
Van Damme, M.P., Robertson, D.M., Diczfalusy, E., 1974. An improved in vitro bioassay method for measuring luteinising hormone (LH) activity using mouse Leydig cell preparations. Acta Endocrinol. 77, 655-671.

Young, A.J., 2009. The causes of physiological suppression in vertebrate societies: a synthesis. In: Reproductive skew in vertebrate societies: proximate and ultimate causes (Hager, R., Jones, C.B., eds). Cambridge: Cambridge University Press.

Young, A.J., Carlson, A.A., Monfort, S.L., Russell, A.F., Bennett, N.C., Clutton-Brock, T., 2006. Stress and the suppression of subordinate reproduction in cooperatively breeding meerkats. Proc. Natl. Acad. Sci. USA 103, 12005-12010.

Young, A.J., Clutton-Brock, T.H., 2006. Infanticide by subordinates influences reproductive sharing in cooperatively breeding meerkats. Biol. Lett. 2, 385-387.

Young, A.J., Monfort, S.L., Clutton-Brock, T.H., 2008. The causes of physiological suppression among female meerkats: a role for subordinate restraint due to the threat of infanticide? Horm. Behav. 53, 131-139.

Young, A.J., Spong, G., Clutton-Brock, T.H., 2007. Subordinate male meerkats prospect for extra-group paternity: alternative reproductive tactics in a cooperative mammal. Proc. R. Soc. B 274, 1603-1609. 


\section{TABLES}

\section{Table 1}

\begin{tabular}{|l|c|c|c|c|c|c|}
\cline { 2 - 7 } \multicolumn{1}{c|}{} & \multicolumn{2}{c|}{$\begin{array}{c}\text { Captures at end of } \\
\text { the DRY period }\end{array}$} & \multicolumn{2}{c|}{$\begin{array}{c}\text { Captures during the } \\
\text { WET period }\end{array}$} & \multicolumn{2}{c|}{$\begin{array}{c}\text { DRY vs WET } \\
\text { period comparison } \\
\text { statistics }\end{array}$} \\
\cline { 2 - 7 } & Median & IQR & Median & IQR & W & P \\
\hline $\begin{array}{l}\text { Baseline LH levels } \\
\text { (miu/ml) }\end{array}$ & 1.5 & $\begin{array}{c}1.00- \\
2.63\end{array}$ & 2.7 & $1.00-3.65$ & 15.0 & 0.39 \\
\hline Post GnRH challenge LH & & $1.25-$ & & $6.30-$ & & \\
levels (miu/ml) & 4.2 & 8.55 & 8.0 & 14.22 & 5.0 & 0.01 \\
\hline GnRH-induced increase in & & $0.25-$ & & $3.05-$ & & \\
LH levels (miu/ml) & 2.1 & 6.90 & 6.5 & 10.23 & 5.0 & 0.01 \\
\hline Body mass (g) & 95 & $89-114$ & 106 & $88-110$ & 10.0 & 0.15 \\
\hline
\end{tabular}

\section{TABLE LEGENDS}

Table 1 - The change in reproductive physiological parameters and body mass among the $\mathrm{n}=11$ subordinate females sampled both at the end of the dry period and at the peak of the subsequent wet period. The final column presents the Wilcoxon matched-pairs test statistics for comparisons between dry and wet period values for each parameter. 


\section{FIGURE LEGENDS}

Figure 1 - Fortnightly rainfall patterns for the study period, indicating the two key trapping periods: one at to the end of the dry period and one at the peak of the wet period.

Figure 2 - Subordinate females exhibited markedly higher post GnRH-challenge LH concentrations during the wet period captures than the dry $\left(\chi^{2}{ }_{1}=5.69 ; \mathrm{P}=0.031\right.$; controlling for a positive effect of body mass: $\left.\chi^{2}=6.97 ; \mathrm{P}=0.019\right)$. This was due principally to a significantly stronger effect of the GnRH challenge on circulating LH concentrations in the wet period than in the dry period (challenge (pre or post) * period (dry or wet) interaction: $\chi^{2}{ }_{1}=8.15 ; \mathrm{P}=0.007$; the effect of the challenge did not vary significantly with body mass: $\left.\chi^{2}{ }_{1}=1.39 ; \mathrm{P}=0.25\right)$. The females' baseline LH concentrations were also slightly, though not significantly, higher in the wet period than the dry (GLMM: $\chi^{2}{ }_{1}=4.19 ; \mathrm{P}=0.052$; baseline $\mathrm{LH}$ was not correlated with body mass: $\left.\chi^{2}{ }_{1}=1.95 ; \mathrm{P}=0.17\right)$. Bars present means $( \pm \mathrm{SE})$.

Figure 3 - (a) Subordinate female urinary cortisol concentrations were significantly higher in the wet period than the dry. Bars present predicted means $( \pm \mathrm{SE})$ after controlling for the maximum time lag since capture and for repeated measures of groups and individuals; see text for statistics. (b) There was no significant interaction between the period - wet (solid line) or dry (dashed line) - and the maximum time lag since capture in determining urinary cortisol concentrations, suggesting that the nature of females' stress responses to capture did not differ significantly between the two periods. 


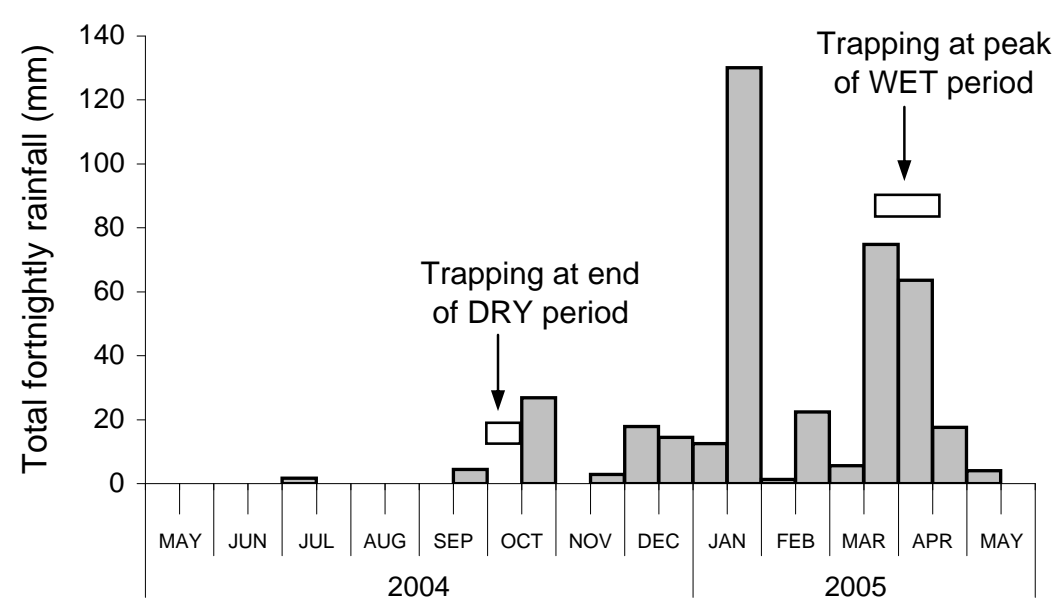

Figure 1 


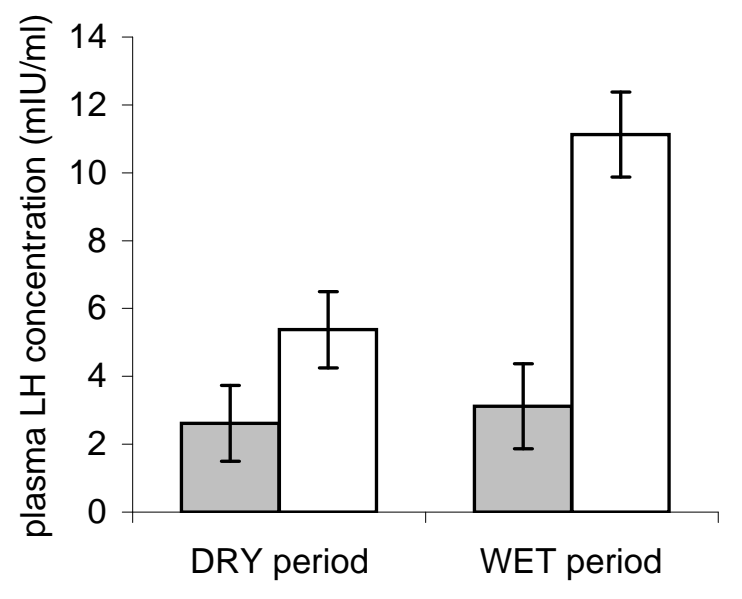

$\square$ Baseline $\square$ Post GnRH Challenge

Figure 2 

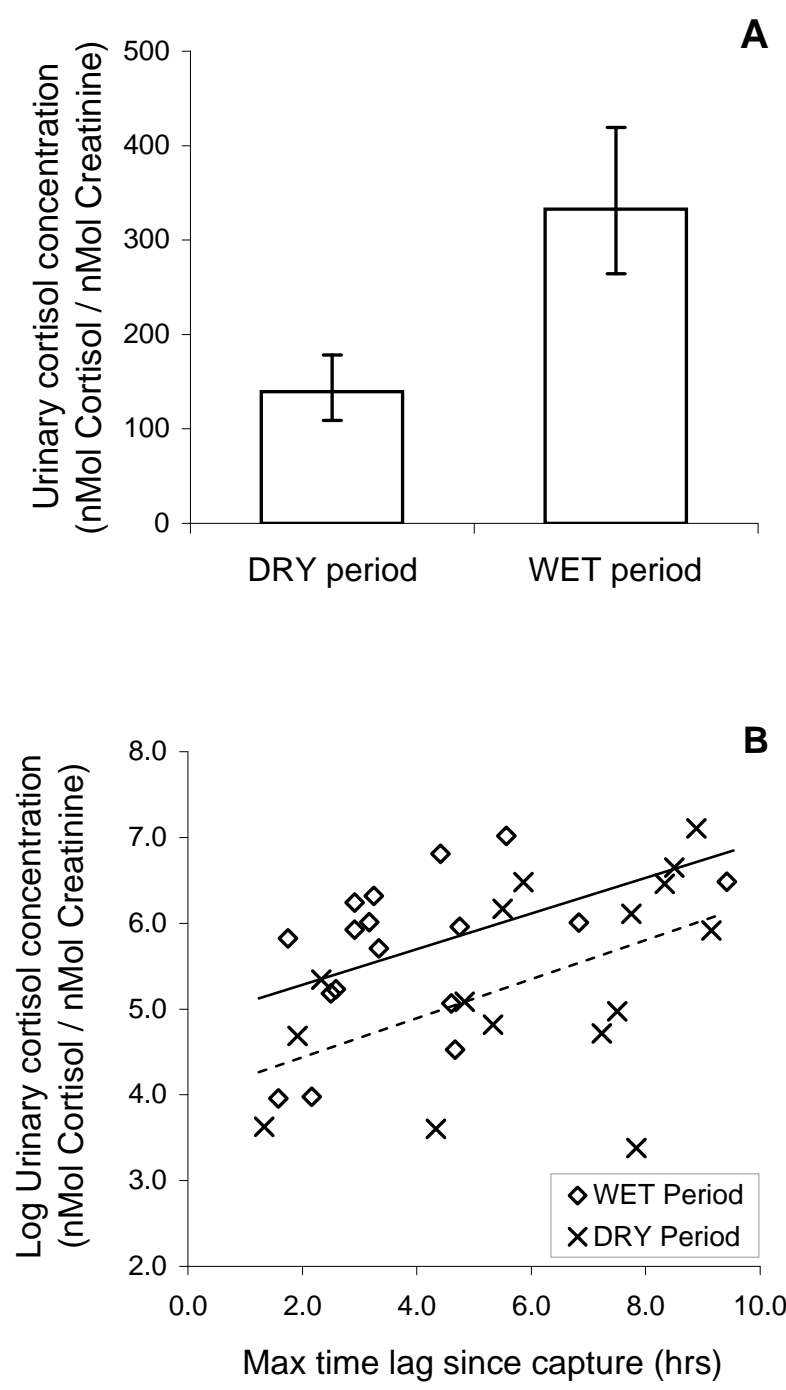

Figure 3 